\title{
Online news media reporting of ketamine as a treatment for depression from 2000 to 2017
}

\author{
Bronagh Gallagher ${ }^{1,2}$, Aaron Neiman ${ }^{3}$, Marie-Claire Slattery ${ }^{1}$ and Declan M. McLoughlin ${ }^{12, *}$ (1) \\ ${ }^{1}$ Department of Psychiatry, Trinity College Dublin, St. Patrick's University Hospital, James Street, Dublin 8, Ireland \\ 2 Trinity College Institute of Neuroscience, Trinity College Dublin, Dublin 2, Ireland \\ ${ }^{3}$ Department of Anthropology, Stanford University, Stanford, CA 94305, USA
}

Objectives: Since the first reporting of ketamine's antidepressant effects in 2000, there has been growing public interest in this novel rapid-acting treatment for depression despite its abuse potential. Online media is an increasingly popular way for the general public to source information. Our objective was to examine how online news outlets have portrayed ketamine as an antidepressant by ascertaining the volume and content of relevant articles and trends over time.

Methods: In this semi-quantitative study, we identified articles regarding ketamine's use in depression from the 30 most popular English-language online news-generating sources over 18 years (2000-2017). Articles were then blindly assessed by 2 independent raters, who analysed the texts by quantifying the presence/absence of 12 content items.

Results: We identified 97 articles, the number of which has increased since the first online news report in 2006. Most (69\%) came from the USA and nearly all correctly stated the indications for ketamine. About half of the most recent articles mentioned abuse potential and $27 \%$ of articles referred to risks of unregulated use of ketamine. Just under $20 \%$ of articles referred to the lack of evidence regarding direct comparisons between ketamine and other currently available antidepressants. There was no difference in the overall level of detail within the articles during the study time period.

Conclusions: Online news media articles have been generally positive about ketamine for treating depression but need to be interpreted with caution as many of them did not discuss negative aspects of ketamine and made unsubstantiated claims about ketamine.

Received 30 December 2020; Revised 22 April 2021; Accepted 18 May 2021

Key words: Antidepressant, glutamate, internet, journalism, mood disorder.

\section{Introduction}

Ketamine is a dissociative anaesthetic drug that was approved by the Food and Drug Administration (FDA) in 1970 and is used routinely in both adult and child anaesthesia. Ketamine's use in psychiatry was actually first reported back in 1973 when it was used as an abreactive agent in a psychiatric inpatient population (Khorramzadeh \& Lotfy, 1973). Since the initial reporting of a single slowly infused sub-anaesthetic dose of ketamine $(0.5 \mathrm{mg} / \mathrm{kg})$ as a rapid-acting antidepressant in 2000 (Berman et al. 2000), there has been growing interest in this potentially novel antidepressant. There have now been multiple clinical trials of both single and serial sub-anaesthetic doses of ketamine that have supported its antidepressant effect (Kishimoto et al. 2016).

Ketamine is a racemic mixture of $\mathrm{R}$ - and S-ketamine (also known as esketamine) enantiomers which in turn

*Address for correspondence: Declan M. McLoughlin, Department of Psychiatry, St. Patrick's University Hospital, James Street, Dublin 8, Ireland.

(Email: d.mcloughlin@tcd.ie) are converted to (R)- and (S)-norketamine and -hydroxynorketamine metabolites. Ketamine acts as a noncompetitive antagonist of the N-methyl-D-aspartate (NMDA) receptor and thus targets the excitatory amino acid neurotransmitter glutamate (Maeng \& Zarate, 2007). However, the precise antidepressant action of ketamine is not yet understood and may involve its metabolites, downstream signalling events and other receptor types, including $\alpha$-amino-3-hydroxy-5methyl-4-isoxazolepropionic acid receptors as well as opioid receptors (Williams et al. 2018; Zanos et al. 2018).

Other methods for administering ketamine to treat depression are being explored, including oral, intranasal, subcutaneous and intramuscular routes (Loo et al. 2016; Andrade, 2017). Ketamine has also been reported to be effective at various sub-anaesthetic doses, suggesting that there is a range of effective doses and routes that merits further investigation with larger participant numbers (Andrade, 2017; Fava et al. 2018). Intranasal esketamine was approved by the FDA in early 2019 for those with treatment resistant depression for regulated use, with detailed dosing and safety information, in conjunction with oral antidepressant 
medication (FDA, 2019). In late 2019, intranasal esketamine was similarly approved also by the European Commission, but it has not been supported by the National Institute for Health and Care Excellence (NICE) in the UK (NICE, 2020). Ketamine itself, however, currently remains unlicensed in both the USA and Europe for treating depression, though there is interest in a specific role for ketamine in the acute management of major depression with suicidal ideation (Wilkinson et al. 2018).

While single and limited serial off-label use of lowdose ketamine under close medical supervision is generally considered to be well-tolerated by patients and reasonably safe (Short et al. 2018), there are substantial concerns about both its unregulated and regulated use for depression (Sanacora et al. 2017; Singh et al. 2017; Schatzberg, 2019). This is because ketamine can produce a mild euphoria as well as dissociative symptoms and sometimes hallucinations. Due to these 'out of body' experiences, ketamine can also be abused and has become a popular 'club drug' since the 1980s (Bokor \& Anderson, 2014). Ketamine can cause some short-term physical side effects including tachycardia and increased blood pressure due to its stimulation of the sympathetic nervous system (Tweed et al. 1972). Chronic, mostly recreational, high-dose ketamine use can cause uropathy and dependency is a concern (Forster \& Harrison, 2012). Of note, however, twiceweekly dosing of intranasal low-dose esketamine has been reported not to be associated with signs of withdrawal or dependence during the 2 weeks after stopping an at least 16-week course (Daly et al. 2019). Approximately 20\% of those who use ketamine recreationally experience urinary symptoms (Winstock et al. 2012) while prolonged use can cause an irreversible cystitis (Tsai et al. 2009). Other potential longer-term side effects of chronic repeated ketamine use include hepatotoxicity and memory impairment (Short et al. 2018) and there is some pre-clinical evidence that it may cause damage to brain white matter ( $\mathrm{Li}$ et al. 2017). There is therefore a risk-benefit ratio to be considered for the safe, supervised and regulated use of ketamine for treating depression as well as a need to identify clear evidence-based indications for its appropriate use in regular clinical practice.

Due to the increased scientific interest in ketamine to treat depression - coupled with concerns about unregulated prescribing and dosing, adequacy of safety monitoring and risk of abuse - we wanted to explore how ketamine was being reported in the public media and do so prior to the commercial developments around intranasal esketamine might shift the focus away from ketamine. Media can have a powerful influence over the general public and has the ability to potentially impact how a medical treatment is viewed. Print news relating to ketamine as a treatment for depression, published in Canada and the USA from 2000 to 2015, was previously reported (Zhang et al. 2017). The authors found that articles published from 2008 onwards were more likely to encourage the use of ketamine in depression, despite a limited evidence base. We widened our search to worldwide online news media and assessed for any similar trends. We decided to examine online news sources due to the increasing trend away from print news and towards digital content (Westlund \& Färdigh, 2015).

\section{Methods \\ Procedures}

Using Amazon.com's Alexa web ranking service (https://www.alexa.com/topsites), we identified the 30 most popular English-language online news sources in July 2018. Popularity was determined by Alexa web ranking, which is based on the average daily time spent on each general site, the number of daily page views per visitor, the percentage of referrals the site receives from web searches and the total number of sites linking to the news site. News aggregators that did not produce original content were excluded. Ethical approval was not sought for this study as it did not involve patients or patient personal information.

Each website was then searched by entering the word 'ketamine' into its native search function (e.g. query.nytimes.com) and limited to content within the date range 1 January 2000- 31 December 2017. There was no minimum word count required for inclusion. For four publications (theguardian.com, news.com.au, timesofindia.indiatimes.com, time.com), the native search algorithm on the site had limited functionality. For these publications, the search was conducted using a bespoke Google Advanced Search (google.com/ advanced_search).

Identified articles were individually reviewed (by author AN) and the articles that selectively discussed ketamine being used as a treatment for depression in humans were included for analysis. The selected articles were catalogued by one researcher (AN), where word count, date and country of each publication were noted. Selected articles were then de-identified by one of the authors (AN) by removing the date, news source and reporter details from each one and converting it to a simple text file. Two raters (BG and CS) separately evaluated each de-identified article according to a 12-item instrument to assess article content and created for the purpose of this study. If there were any discrepancies between the presence or absence of any of the 12 items, the two raters met to resolve this disagreement by reviewing the articles again to reassess the item in 
question. If an agreement could not be reached, a third party could be consulted (DMM).

The article assessment instrument consisted of 12 binary questions, aimed to capture some specific details about ketamine and its role in treating depression: indications for use; onset, duration and mechanism of action; comparison with conventional antidepressants; side effects and potential for misuse; regulatory matters; the depth of reporting by inclusion of expert and patient opinion and experience. These questions were chosen to reflect important aspects of clinical practice in order to inform interested readers. Raters noted the presence or absence of the following in each article.

1. Did the article discuss the potential clinical indications for ketamine when treating depression, for example, treatment resistance, suicidal ideation?

2. Did the article describe the rapid onset of ketamine's antidepressant effects?

3. Did the article describe the duration of ketamine's antidepressant effects?

4. Did the article discuss ketamine's potential mechanism of action?

5. Did the article discuss the lack of long-term evidence that ketamine is better than standard antidepressants?

6. Did the article describe subjective effects of ketamine such as dissociation, sedation and euphoria?

7. Did the article mention ketamine's potential physical side effects?

8. Did the article mention ketamine's abuse or addictive potential?

9. Was there a discussion of the presence/absence of any formal guidance on ketamine, for example, FDA, European Union approval?

10. Did the article discuss the risks of unregulated or unsupervised use of ketamine?

11. Was there a researcher consulted or quoted?

12. Was there a patient quoted?

Each question was then given a score of 1 if answered 'yes' and 0 if answered 'no'. Each of the 97 articles was then given a score out of 12 which reflected the level of detail within the article (Detail Score), with higher scores representing a more comprehensive overview of ketamine in the treatment of depression.

\section{Data analysis}

Statistical analyses were performed using SPSS version 24.0 (IBM Corporation, NY, USA). For each of the 12 article content questions, the articles were grouped into four distinct time frames, each spanning an equal 3-year period: 2006-2008, 2009-2011, 2012-2014 and 20152017. For the proportions that answered yes or no to each of the 12 questions, we used either a chi-squared or Fisher's exact test to assess for differences between the four time frames. An analysis of variance was used to assess differences between mean Detail Scores of each of the four time frames. A $p$ value $<0.05$ was deemed statistically significant.

\section{Results}

A total of 2809 articles, from the top 30 English-language online news websites (see Table 1), were identified using the search method between 1 January 2000 and 31 December 2017. Of the 2809 articles, 2569 were excluded for irrelevance, meaning the article did not mention ketamine for antidepressant purposes (see Fig. 1). Further exclusions were made if the article was not a news item but an opinion piece or personal essay $(n=18)$, and if the article was a duplicate publication syndicated from another source $(n=53)$. Of the remaining 169 articles, 72 were further excluded because the content was primarily about the use of ketamine for other psychiatric purposes besides depression treatment $(n=8)$, ketamine's use as an antidepressant was only briefly mentioned (i.e. referred to in only one sentence) in the article $(n=49)$, the article was primarily about the legal status of ketamine $(n=7)$ or the article was primarily about the financial aspects of depression pharmaceuticals $(n=8)$. The content of the remaining 97 articles was analysed.

The 30 websites were broken down into regions as follows: USA $(n=19)$, India $(n=4)$, the United Kingdom $(n=3)$, Australia $(n=1)$, China $(n=1)$, Germany $(n=1)$ and Canada $(n=1)$ (Table 1$)$. The vast majority $(67 / 97=69.1 \%)$ of relevant news articles published online between 2000 and 2017 were from the USA. UK and India, respectively, had 16 (16.5\%) and $10(10.3 \%)$ articles each. Both Canada and Australia had just two $(2.1 \%)$ articles each.

Ketamine's novel antidepressant effect was first reported in 2000 (Berman et al. 2000). However, the first online news article relating to ketamine as an antidepressant was not published until 2006 (Fig. 2). This coincided with the publication of a randomised crossover trial $(n=18)$ that confirmed the rapid-onset antidepressant effect of ketamine (Zarate et al. 2006). The results of this small but influential trial were very positive, with $71 \%$ of patients treated with a single subanaesthetic dose of ketamine meeting antidepressant response criteria 1 day after the infusion. The highest number of articles published was in 2014 and 2017 (both $n=19$ ). Most relevant articles have been published from 2012 onwards.

De-identified articles were evaluated by two blinded raters using the 12-item instrument created for this study. Of 1164 pairs of observations for the 12 binary rating items, there was an interrater agreement of $94.6 \%$ and 63 discrepancies were noted. The two raters (BG and CS) met to resolve these discrepancies and 
Table 1. The top 30 English-language news-generating websites and the number of articles included in the analysis

\begin{tabular}{|c|c|c|c|}
\hline Rank & Online news site & Web address & Number of articles analysed per site \\
\hline 1 & $\mathrm{CNN}$ & cnn.com & 1 \\
\hline 2 & New York Times & nytimes.com & 5 \\
\hline 3 & The Guardian & theguardian.com & 9 \\
\hline 4 & Washington Post & washingtonpost.com & 2 \\
\hline 5 & $\mathrm{BBC}$ & bbc.co.uk & 2 \\
\hline 6 & Forbes & forbes.com & 6 \\
\hline 7 & Huffington Post & huffingtonpost.com & 4 \\
\hline 8 & Fox News & foxnews.com & 6 \\
\hline 9 & Bloomberg & bloomberg.com & 3 \\
\hline 10 & The Times of India & indiatimes.com & 7 \\
\hline 11 & USA Today & usatoday.com & 1 \\
\hline 12 & Reuters & reuters.com & 5 \\
\hline 13 & Wall Street Journal & wsj.com & 2 \\
\hline 14 & Time & time.com & 10 \\
\hline 15 & New York Post & nypost.com & 2 \\
\hline 16 & The Atlantic & theatlantic.com & 2 \\
\hline 17 & LA Times & latimes.com & 4 \\
\hline 18 & US News & usnews.com & 1 \\
\hline 19 & The Indian Express & indianexpress.com & 1 \\
\hline 20 & ABC News & abcnews.go.com & 1 \\
\hline 21 & NBC News & nbcnews.com & 1 \\
\hline 22 & CBS News & cbsnews.com & 4 \\
\hline 23 & News.com.au & news.com.au & 2 \\
\hline 24 & China Daily & chinadaily.com.cn & 0 \\
\hline 25 & The Hindu & thehindu.com & 0 \\
\hline 26 & SF Gate & sfgate.com & 11 \\
\hline 27 & The Daily Beast & thedailybeast.com & 1 \\
\hline 28 & CBC news & cbc.ca/news & 2 \\
\hline 29 & DW news & dw.com & 0 \\
\hline 30 & Hindustan Times & hindustantimes.com & 2 \\
\hline
\end{tabular}

came to a mutual agreement on the presence or absence of each item. Figure 3 illustrates the breakdown of each question according to the time frame.

Almost all the articles $(95 / 97 ; 97.9 \%)$ stated the clinical indications for ketamine when treating depression such as suicidal ideation or treatment resistance. The vast majority commented on its rapid onset of antidepressants effect $(72 / 97 ; 74.2 \%)$; however, less than half remarked on ketamine's relatively short duration of action $(38 / 97 ; 39.2 \%)$.

With regard to ketamine's mechanism of action, it has been discussed less in recent years and there was a significant difference in the frequency of reporting between the 2015-2017 period and the 2012-2014 and 2009-2011 periods $(p<0.005)$. Interestingly, only a small proportion of the articles $(19 / 97 ; 19.6 \%)$ discussed the lack of long-term evidence of ketamine's comparison to currently available antidepressants. Mention of ketamine's abuse potential in online media has gradually risen over time to over $50 \%(21 / 40)$ of articles in 2015-2017 from 0\% (0/9) in the 2009-2011 period $(p=0.010)$.

Only $26.8 \%$ (26/97) of articles mentioned the risks of unregulated use of ketamine as a treatment for depression. Similarly, rates of articles discussing recommendations and formal guidance on how ketamine should be used as an antidepressant treatment were relatively low at $43.3 \%(42 / 97)$.

The majority of the articles quoted a researcher (83/ $97 ; 85.6 \%)$, though most did not quote a patient $(n=14$ / $97 ; 14.4 \%$ ). Physical side effects of ketamine and subjective effects experienced from the drug were mentioned in $62.9 \%(61 / 97)$ and $58.8 \%(57 / 97)$, respectively, of the articles and this proportion remained constant throughout the four time frames.

Each article was provided with a Detail Score out of 12 , depending on how many content items were points of discussion in the article. We calculated mean Detail Scores and 95\% confidence intervals (CI) for each 3-year time frame. An analysis of variance showed no 


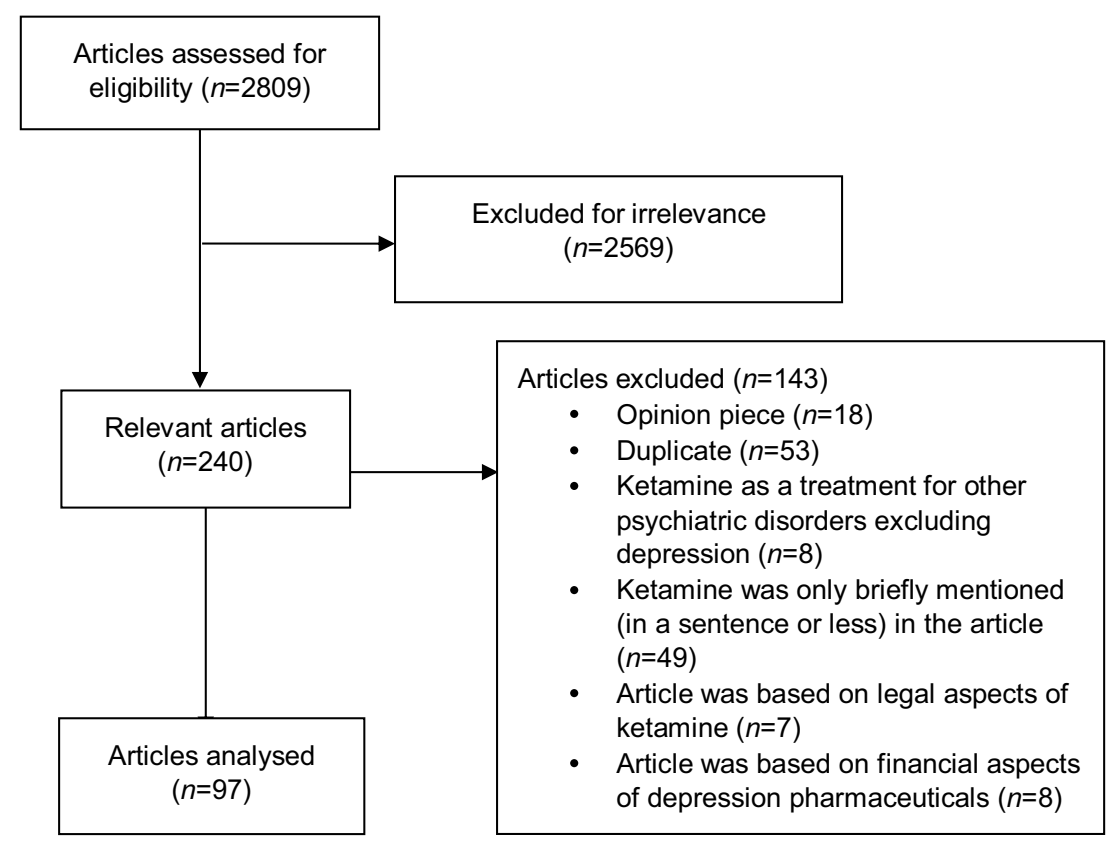

Fig. 1. A flow chart showing the reasons why various articles were excluded in this study.

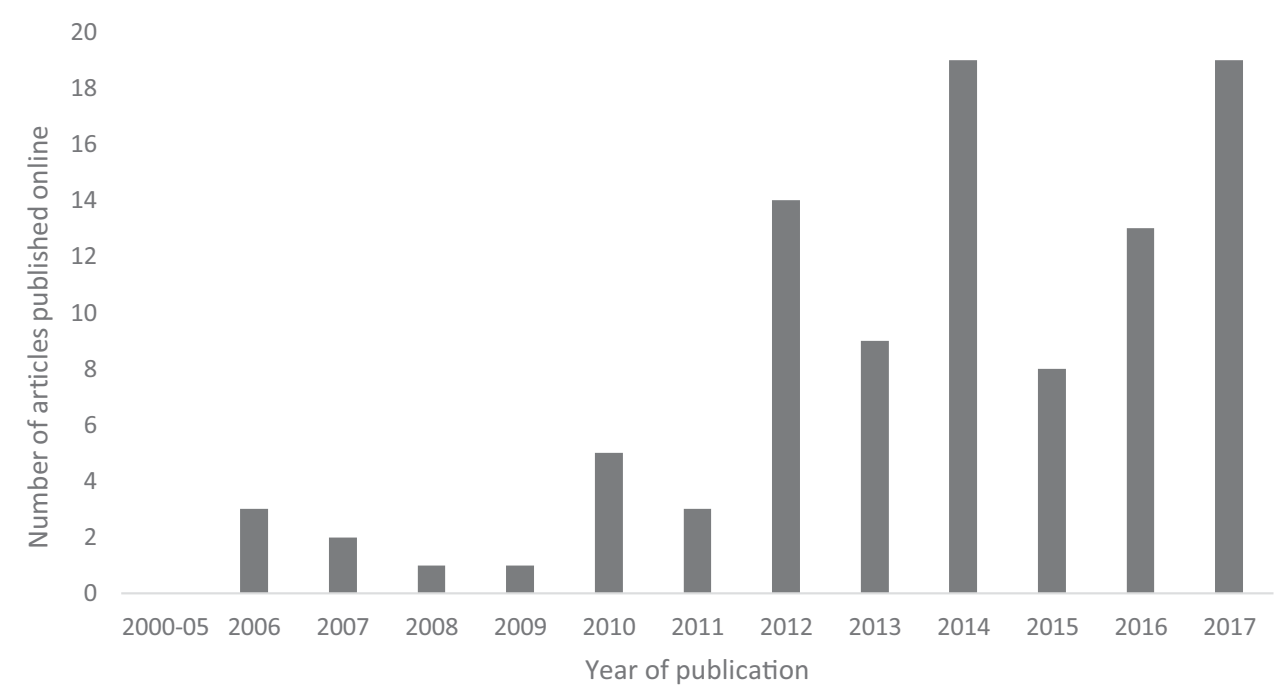

Fig. 2. Number of ketamine online news articles published each year from 2000 to 2017.

statistically significant differences between the mean scores for the four time frames $(F(3,93)=0.488$, $p=0.691)$. Mean $(95 \% \mathrm{CI})$ scores ranged from 5.33 to 6.29 (95\% CI 3.70-8.30) (Fig. 4), suggesting that the level of detail provided in these articles has remained constant throughout the examined time period.

\section{Discussion}

To our knowledge, this is the first study that has analysed online news media sources relating to ketamine's antidepressant effect. We examined the 30 most popular English-language online news-generating sources and explored potential trends. The first relevant online news item was in 2006 with a substantial increase maintained since 2012. Further increases might be expected after 2019 following the FDA's and European Commission's approval of intranasal esketamine.

We looked at how ketamine was portrayed in the media during the study period to see if there were any obvious trends. In terms of the various issues we examined, the level of detail within the articles has remained consistent from 2006 to 2017. As expected, 


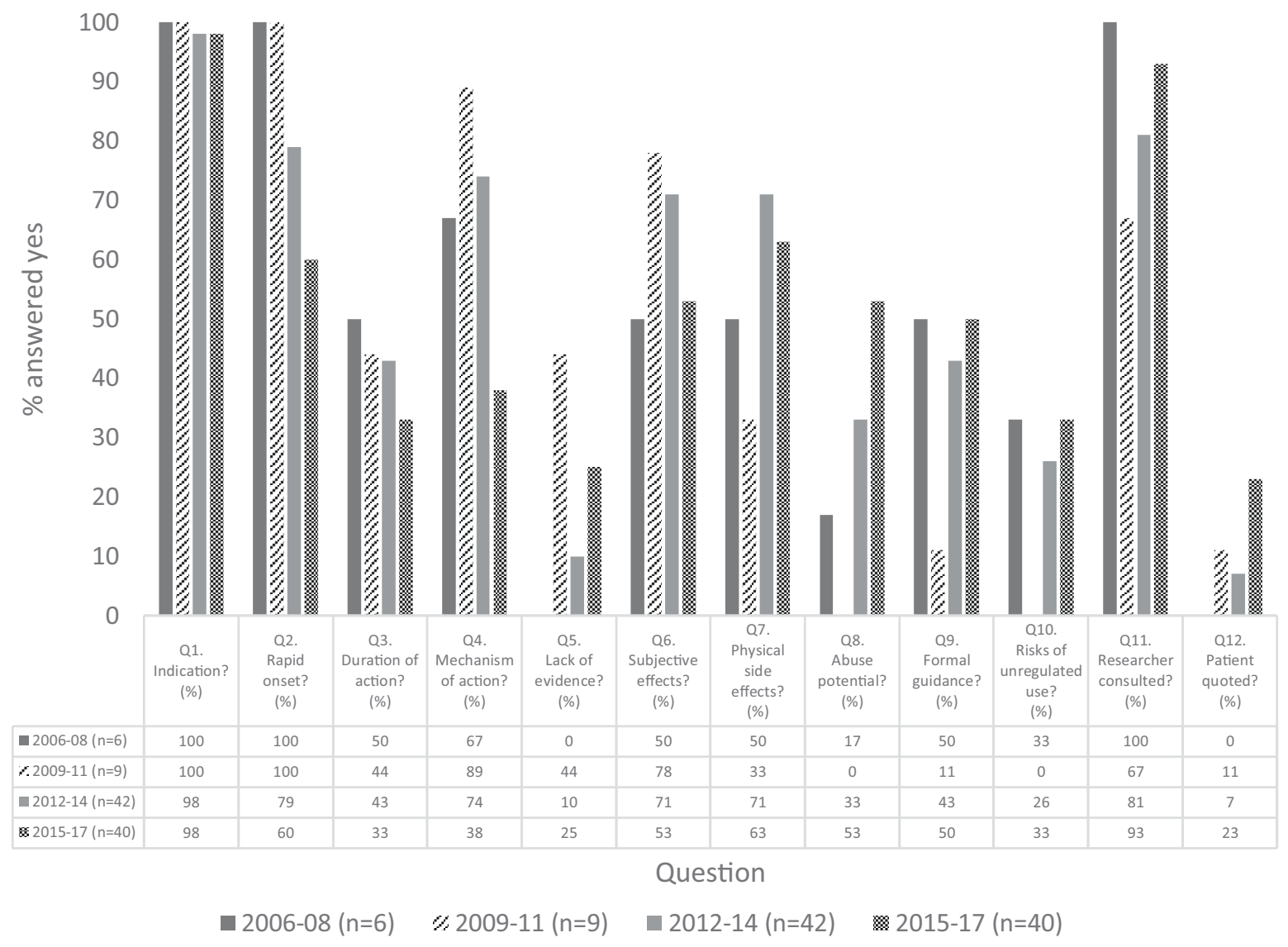

Fig. 3. Breakdown of content analysis by each 3-year period. The y-axis shows the proportion (\%) of 'yes' answers to the 12 content items within each 3-year period.

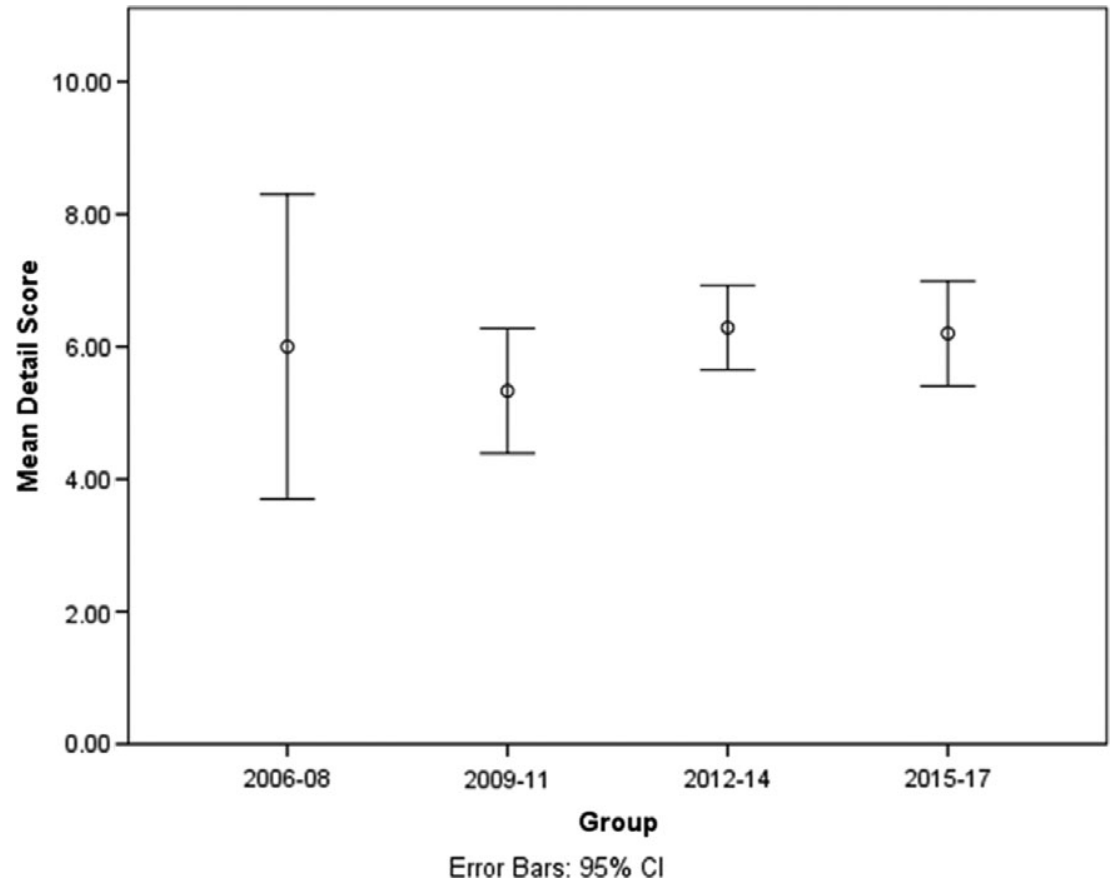

Fig. 4. The mean scores (with $95 \%$ confident intervals) of the articles for each of the four time frames indicating the level of detail within the articles. 
nearly all articles described the clinical indications for ketamine and most referred to its rapid onset of action. Reporting on formal guidance for using ketamine and risks of unregulated use was variable with less than half of the reviewed articles addressing these types of governance issues. Researchers were frequently quoted, though only a few articles consulted with patients. Clinical concerns about ketamine and its potential for abuse were discussed in few of the articles as was the limited evidence of ketamine's comparison to other antidepressants.

In general, sub-anaesthetic doses of ketamine are deemed to be safe and well tolerated. However, physical and psychotomimetic side effects are not uncommon (Short et al. 2018). It is important, therefore, that at least some of these potential adverse effects of ketamine are brought to the public's attention. These were mentioned in just over half the articles analysed.

Ketamine's mechanism of action was discussed less within the reviewed articles in more recent years. Perhaps this is related to multiple emerging theories about how ketamine exerts its antidepressant effects and uncertainty on this issue. These include the NMDA glutamate hypothesis resulting in downstream neuroplasticity (Maeng \& Zarate, 2007) as well as the more recent opioid hypothesis (Williams et al. 2018).

To date, there have been no published trials that have directly compared ketamine to standard antidepressants or other therapies. Only 20\% (19/97) of the articles acknowledged this with some articles even claiming that ketamine's antidepressant effects are superior to currently available antidepressants. Such claims should be interpreted with caution as there are no published trials to date that have specifically tested this. Randomised clinical trials so far have compared ketamine to either placebo (usually saline) or an active comparator such as midazolam. Midazolam is a short acting benzodiazepine that has a similar half-life and sedative effects as low-dose ketamine and therefore may help with blinding (Grunebaum et al. 2018). However, midazolam is not routinely used as an antidepressant.

Limitations of our study are that we only examined English-language media and that we limited our search and analyses to the top 30 online news sources. Inclusion of online news media in other languages would have increased the sample size and may have allowed analysis of regional differences. The use of an un-validated instrument developed for this study is an additional limitation. We limited our search to the end of 2017. This was to avoid the possible impact of news reporting on intranasal esketamine and potential confusion with racemic, mostly intravenous, ketamine for depression. We are planning a separate follow-up study, following the approval of esketamine in the treatment of treatment resistant depression, which will explore the impact of this on news media reporting.

Overall, our findings on international online new media reporting of ketamine for depression parallel those reported for North American print media (Zhang et al. 2017). Currently, intravenous ketamine is not licenced for treating depression in the USA or Europe, though this may change in the near future. In many countries around the world, ketamine is a controlled substance due to its risk for abuse (Nickerson \& Attaran, 2015). It is important to note that, to date, there has not been evidence to suggest that ketamine causes dependency after twice-thrice weekly intravenous dosing over a number of weeks (Singh et al. 2016). The potential longer-term side effects of frequent sub-anaesthetic doses of ketamine are as yet undetermined because research in this area has so far been limited. Although there is no evidence of physical dependence, ketamine can be psychologically addictive (Bokor \& Anderson, 2014). It is therefore important that, in addition to its potential benefits, we highlight the side effects and addictive potential when discussing ketamine with the general public. The risks of unregulated use of ketamine in psychiatry should also be highlighted to the general public.

Based on the finding described above, we would recommend interpreting news media reports of ketamine for depression with caution. Reporters should also have an awareness of the impact of their articles on the views of the general public and how influential they can be.

\section{Conflicts of interest}

Declan M. McLoughlin has received speaker's honoraria from Mecta and Otsuka and an honorarium from Janssen for participating in an esketamine advisory board meeting. The other authors report no conflicts of interest.

\section{Ethical standards}

The authors assert that all procedures contributing to this work comply with the ethical standards of the relevant national and institutional committee on human experimentation with the Helsinki Declaration of 1975, as revised in 2008.

\section{Financial support}

This research received no specific grant from any funding agency, commercial or not-for-profit sectors. 


\section{References}

Andrade C (2017). Ketamine for Depression, 4: In What Dose, at What Rate, by What Route, for How Long, and at What Frequency? Journal of Clinical Psychiatry 78, e852-e857.

Berman RM, Cappiello A, Anand A, Oren DA, Heninger GR, Charney DS, Krystal JH (2000). Antidepressant effects of ketamine in depressed patients. Biological Psychiatry 47, 351-354.

Bokor G, Anderson PD (2014). Ketamine: an update on its abuse. Journal of Pharmacy Practice 27, 582-586.

Daly EJ, Trivedi MH, Janik A, Li H, Zhang Y, Li X, Lane R, Lim P, Duca AR, Hough D, Thase ME, Zajecka J, Winokur A, Divacka I, Fagiolini A, Cubala WJ, Bitter I, Blier P, Shelton RC, Molero P, Manji H, Drevets WC, Singh JB (2019). Efficacy of Esketamine Nasal Spray Plus Oral Antidepressant Treatment for Relapse Prevention in Patients With Treatment-Resistant Depression: A Randomized Clinical Trial. JAMA Psychiatry 76, 893-903.

Fava M, Freeman MP, Flynn M, Judge H, Hoeppner BB, Cusin C, Ionescu DF, Mathew SJ, Cahng LC, Iosifescu DV, Murrough J, Debattista C, Schatzberg AF, Trivedi MH, Jha MK, Sanacora G, Wilkinson St, Papakostas GI (2018). Double-blind, placebo-controlled, dose-ranging trial of intravenous ketamine as adjunctive therapy in treatment-resistant depression (TRD). Molecular Psychiatry 25, 1592-1603.

FDA. (2019). U.S Food and Drug Administration [online]. Available at: https://www.fda.gov/news-events/pressannouncements/fda-approves-new-nasal-spraymedication-treatment-resistant-depression-available-onlycertified.

Forster JA, Harrison SC (2012). Ketamine uropathy: rising to the challenges of a new condition. BJU International 109, 1277-1278.

Grunebaum MF, Galfalvy HC, Choo TH, Keilp JG, Moitra VK, Parris MS, Marver JE, Burke AK, MIlak MS, Sublette ME, Oquendo MA, Mann JJ (2018). Ketamine for Rapid Reduction of Suicidal Thoughts in Major Depression: A Midazolam-Controlled Randomized Clinical Trial. American Journal Psychiatry 175, 327-335.

Khorramzadeh E, Lotfy AO (1973). The use of ketamine in psychiatry. Psychosomatics 14, 344-346.

Kishimoto T, Chawla JM, Hagi K, Zarate CA, Kane JM, Bauer M, Correll CU (2016). Single-dose infusion ketamine and non-ketamine N-methyl-d-aspartate receptor antagonists for unipolar and bipolar depression: a meta-analysis of efficacy, safety and time trajectories. Psycholofical Medicine 46, 1459-1472.

Li Q, Shi L, Lu G, Yu HL, Yeung FK, Wong NK, Sun L, Liu K, Yew D, Pan F, Wang DF, Sham PC (2017). Chronic Ketamine Exposure Causes White Matter Microstructural Abnormalities in Adolescent Cynomolgus Monkeys. Frontiers in Neuroscience 11, 285.

Loo CK, Galvez V, O'Keefe E, Mitchell PB, Hadzi-Pavlovic D, Leyden J, Harper S, Somogyi AA, Lai R, Weickert CS, Glue P (2016). Placebo-controlled pilot trial testing dose titration and intravenous, intramuscular and subcutaneous routes for ketamine in depression. Acta Psychiatrica Scandinavica 134, 48-56.

Maeng S, Zarate CA Jr. (2007). The role of glutamate in mood disorders: results from the ketamine in major depression study and the presumed cellular mechanism underlying its antidepressant effects. Current Psychiatry Reports 9, 467-474.

NICE (NationalInstitute For Health And Care Excellence) (2020) Esketamine for treatment-resistant depression (ID1414). https: / /www.nice.org.uk/guidance/ indevelopment/gid-ta10371.

Nickerson JW, Attaran A (2015). The Commission on Narcotic Drugs' attempt to restrict ketamine. Lancet 385, e19.

Sanacora G, Frye MA, McDonald W, Mathew SJ, turner MS, Schatzberg AF, Summergrad P, Nemeroff CB, American Psychiatric Association Council of Research Task Force on Novel Biomarkers and Treatments (2017). A Consensus Statement on the Use of Ketamine in the Treatment of Mood Disorders. JAMA Psychiatry 74, 399-405.

Schatzberg AF (2019). A Word to the Wise About Intranasal Esketamine. American Journal of Psychiatry 176, 422-424.

Short B, Fong J, Galvez V, Shelker W, Loo CK (2018). Sideeffects associated with ketamine use in depression: a systematic review. Lancet Psychiatry 5, 65-78.

Singh I, Morgan C, Curran V, Nutt D, Schlag A, McShane R (2017). Ketamine treatment for depression: opportunities for clinical innovation and ethical foresight. Lancet Psychiatry 4, 419-426.

Singh JB, Fedgchin M, Daly EJ, De Boer P, Cooper K, Murrough JW, Sanacora G, Shelton RC, Kurian B, Winokur A, Fava A, Manji H, Drevets WC, Van Neuten L (2016). A Double-Blind, Randomized, PlaceboControlled, Dose-Frequency Study of Intravenous Ketamine in Patients With Treatment-Resistant Depression. American Journal of Psychiatry 173, 816-826.

Tsai TH, Cha TL, Lin CM, Tsao CW, Tang SH, Chuang FP, Wu ST, Sun GH, Yu DS, Chang SY (2009). Ketamineassociated bladder dysfunction. International Journal of Urology 16, 826-829.

Tweed WA, Minuck M, Mymin D (1972). Circulatory responses to ketamine anesthesia. Anesthesiology 37, 613-619.

Westlund O, Färdigh MA (2015). Accessing the news in an age of mobile media: tracing displacing and complementary effects of mobile news on newspapers and online news. Mobile Media \& Communication 3, 53-74.

Wilkinson ST, Ballard ED, Bloch MH, Mathew SJ, Murrough JW, Feder A, Sos P, Wang G, Zarate CA, Sanacora G (2018). The Effect of a Single Dose of Intravenous Ketamine on Suicidal Ideation: A Systematic Review and Individual Participant Data Meta-Analysis. American Journal of Psychiatry 175, 150-158.

Williams NR, Heifets BD, Blasey C, Sudheimer K, Pannu J, Pankow H, Hawkins J, Birnbaum J, Lyons DM, Rodriguez CI, Schatzberg AF (2018). Attenuation of Antidepressant Effects of Ketamine by Opioid Receptor 
Antagonism. American Journal of Psychiatry 175, 1205-1215.

Winstock AR, Mitcheson L, Gillatt DA, Cottrell AM (2012).

The prevalence and natural history of urinary symptoms among recreational ketamine users. BJU International 110, $1762-1766$.

Zanos P, Thompson SM, Duman RS, Zarate CA,

Gould TD (2018). Convergent Mechanisms Underlying Rapid Antidepressant Action. CNS Drugs 32, 197-227.
Zarate CA Jr, Singh JB, Carlson PJ, Brutsche NE, Ameli R, Luckenbaugh DA, Charney DS, Manji HK (2006). A randomized trial of an N-methyl-D-aspartate antagonist in treatment-resistant major depression. Archives of General Psychiatry 63, 856-864.

Zhang MW, Hong YX, Husain SF, Harris KM, Ho RC (2017). Analysis of print news media framing of ketamine treatment in the United States and Canada from 2000 to 2015. PLoS One 12, e0173202. 